
Not for reproduction, distribution or commercial use.

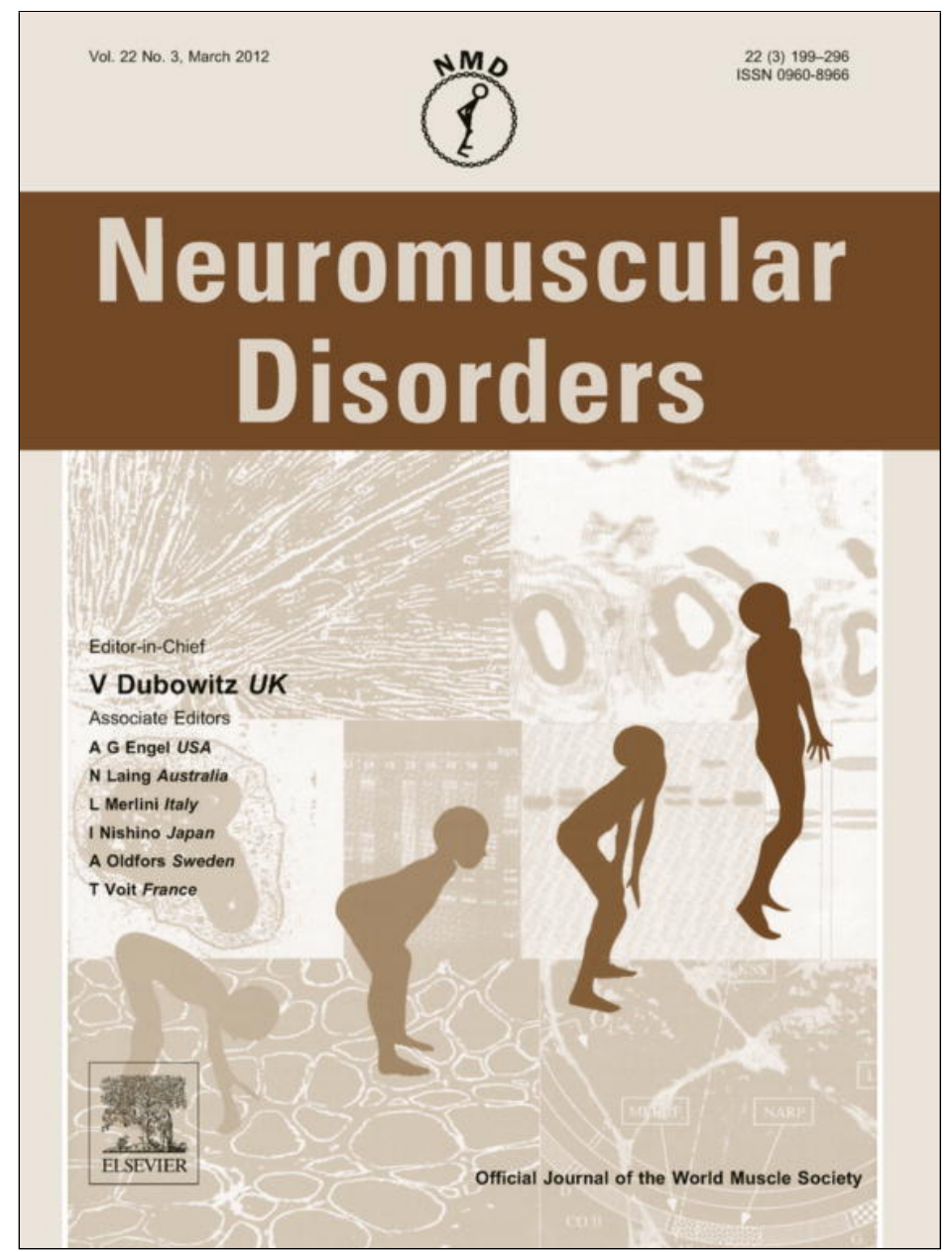

This article appeared in a journal published by Elsevier. The attached copy is furnished to the author for internal non-commercial research and education use, including for instruction at the authors institution and sharing with colleagues.

Other uses, including reproduction and distribution, or selling or licensing copies, or posting to personal, institutional or third party websites are prohibited.

In most cases authors are permitted to post their version of the article (e.g. in Word or Tex form) to their personal website or institutional repository. Authors requiring further information regarding Elsevier's archiving and manuscript policies are encouraged to visit:

http://www.elsevier.com/copyright 


\title{
High cardiovascular morbidity and mortality in myofibrillar myopathies due to DES gene mutations: a 10-year longitudinal study
}

\author{
Karim Wahbi $^{\mathrm{a}, *}$, Anthony Béhin ${ }^{\mathrm{a}}$, Philippe Charron ${ }^{\mathrm{b}, \mathrm{c}}$, Murielle Dunand ${ }^{\mathrm{d}}$, \\ Pascale Richard $^{\mathrm{e}}$, Christophe Meune ${ }^{\mathrm{f}}$, Patrick Vicart ${ }^{\mathrm{g}}$, Pascal Laforêt ${ }^{\mathrm{a}}$, Tanya Stojkovic ${ }^{\mathrm{a}}$, \\ Henri Marc Bécane ${ }^{\mathrm{a}}$, Thierry Kuntzer ${ }^{\mathrm{d}}$, Denis Duboc ${ }^{\mathrm{f}}$ \\ ${ }^{a}$ Institut de Myologie, Hôpital Pitié-Salpêtrière, APHP, Paris, France \\ ${ }^{\mathrm{b}}$ UPMC Université Paris 6, France \\ ${ }^{\mathrm{c}}$ AP-HP, Département de Génétique, Hôpital Pitié-Salpêtrière, INSERM UMR956, Paris, France \\ ${ }^{\mathrm{d}}$ Service de Neurologie, Centre Hospitalier Universitaire Vaudois, Université de Lausanne, 1011, Lausanne, Switzerland \\ ${ }^{\mathrm{e}}$ Assistance Publique- Hôpitaux de Paris, UF Cardiogénétique et Myogénétique Moléculaire, Service de Biochimie, Hôpital Pitié-Salpêtrière, APHP, \\ Paris, France \\ ${ }^{\mathrm{f}}$ Service de Cardiologie, Hôpital Cochin, APHP, Université Paris Descartes, Paris, France \\ ${ }^{\mathrm{g}}$ Equipe Stress et Pathologies du Cytosquelette, Unité de Biologie Fonctionnelle et Adaptative, Université Paris Diderot-Paris 7/CNRS, EAC 4413 Paris, \\ France
}

Received 22 June 2011; received in revised form 29 September 2011; accepted 26 October 2011

\begin{abstract}
To determine incidence and type of major cardiac adverse events in patients with mutated desmin $(D E S)$ gene, we retrospectively reviewed baseline medical information, and examined the long-term outcomes of 28 DES patients (17 men, baseline mean age $=37.7 \pm 14.4$ years $[\min =9$, $\max =71]$ ) from 19 families. Baseline studies revealed skeletal muscle involvement in 21 patients and cardiac abnormalities in all but one patient. Over a mean follow-up of $10.4 \pm 9.4$ years [ $\min =1$, $\max =35$ ], cardiac death occurred in three patients, death due to cardiac comorbidities in two, one or more major cardiac adverse events in 13 patients. Among the 19 patients with mild conduction defects at baseline, eight developed high-degree conduction blocks requiring permanent pacing. Cardiac involvement was neither correlated with the type of DES mutation nor with the severity of skeletal muscle involvement. Our study underscores that in $D E S$ patients in-depth cardiac investigations are needed to prevent cardiac conduction system disease.
\end{abstract}

(C) 2011 Elsevier B.V. All rights reserved.

Keywords: Desminopathy; Pacemaker; Cardiomyopathy

\section{Introduction}

Desmin is a cytoskeletal protein which forms intermediate filaments and interacts with other proteins to create an intracytoplasmic network. This protein is known to play an important role in the attachment, stabilization, and force

\footnotetext{
* Corresponding author. Address: Institut de Myologie, Hôpital PitiéSalpêtrière, 47-83, boulevard de l'Hôpital - 75651, Paris Cedex 13, France. Tel.: +33 (0)1 421637 75; fax: +33(0)1 42163793 .

E-mail address: karim.wahbi@psl.aphp.fr (K. Wahbi).
}

transmission of the sarcomeres in skeletal and cardiac muscle cells [1]. Desminopathies encompass the genetic diseases caused by mutations in the human desmin gene $(D E S)$ on chromosome $2 \mathrm{q} 35$ [2,3]. In those rare diseases, clinical manifestations include familial or sporadic cardiomyopathies [4-7], and skeletal muscle weakness with myofibrillar changes, or both of them [8-15].

Dilated, hypertrophic or restrictive cardiomyopathies, and arrhythmias have all been reported in patients with desminopathy [8-13], however the prognostic significance of these abnormalities is not well known due to absence of long-term outcome studies. Therefore, no consensus 
has been reached regarding the best diagnostic and therapeutic strategies in patients with desminopathy.

The objectives of this longitudinal retrospective study were to (a) analyze clinical information based on standardized skeletal muscle and cardiac investigations in consecutive patients suffering from DES mutations, and (b) examine their long-term clinical outcomes with particular focus on the incidence and type of major cardiac adverse events (AEs) during follow-up.

\section{Patients and methods}

We retrospectively identified the consecutive patients with DES mutations who, between 1981 and 2009, were referred to the neurology, cardiology or genetics units of the Pitié Salpêtrière, Cochin and Lausanne University Hospitals, for evaluation and treatment of skeletal muscle or cardiac disease manifestations, or for systematic family screening. The standardized muscle and cardiac studies they underwent at diagnosis were repeated atleast every 2 years. The baseline cardiac and muscle assessments, as well as cardiac complications which occurred during follow-up (see cardiac studies, below), were retrospectively entered in a dedicated database.

\subsection{Standard protocol approvals, registrations, and patient consents}

The study was approved by the ethics committee of the participating institutions, and the patients granted their informed consent to contribute their medical information to this analysis.

\subsection{Genetic testing}

Blood samples were collected after obtaining written informed consent from all patients. Genomic DNA was extracted from peripheral blood lymphocytes using the Genomic-Mega-Extractor (EXTRAGENE) and PROMEGA chemistry according to the manufacturer's procedures.

A series of primer pairs (available on request) was designed to amplify the 9 coding exons and flanking intronic sequences of $D E S$ based on the published sequence (GenBank accession number NG_008043.1). After purification, the PCR products were sequenced on both strands using the ABI BigDye ${ }^{\circledR}$ Terminator v3.2 Kit (Applied Biosystems, Foster, CA) and run on an ABI3730 device (Applied Biosystems). SeqScape ${ }^{\circledR}$ Software was used to facilitate data analysis and mutation identification followed by visual inspection of individual sequencing traces. Detected variants in a sample were confirmed by an independent PCRs and sequencing runs. An analysis with Alamut Software (Interactive Biosoftware, Rouen, France) was systematically conducted to predict possible impact on any amino acid substitution. Genetic variants were annotated according to the protein and cDNA reference sequences
(UniProtKB/Swiss-Prot P17661 and NM_001927.3). Several criteria were used to determine if a new variant is a rare polymorphism or a potential disease causing mutation; (i) the conservation of the nucleotide and the aminoacid among species and isoforms, (ii) the absence of the variant in control chromosomes, (iii) the modification of charge of the mutated residue, (iv) the cosegregation in affected individuals of the same family.

\subsection{Muscle investigations}

At the time of diagnosis, all probands underwent (a) a detailed physical examination by a neurologist specialized in muscle disorders, (b) measurement of serum creatine kinase level (CK), and (c) a muscle biopsy. All probands' relatives were investigated according to the same protocol, but muscle biopsy was not systematically performed.

\subsection{Cardiac studies}

The cardiac studies included electrocardiograms (ECG) and echocardiograms [14] recorded at baseline and during follow-up in all patients. Patients whose ECG revealed the presence of conduction abnormalities underwent 24-h ambulatory ECG. Coronary angiography was performed in patients $\geqslant 40$ years of age and evidence of left ventricular dysfunction, to exclude the presence of ischemic cardiomyopathy.

Cardiac involvement was considered present in patients with (a) abnormalities of impulse propagation, defined by a PR interval $>200$ min or a QRS duration $>110 \mathrm{~min}$, (b) sustained atrial arrhythmias, (c) non-sustained or sustained ventricular tachycardia, or (d) left (LV) or right ventricular (RV) dilation, hypertrophy or systolic dysfunction. Cardiac adverse events (AEs) were classified as major when the following conditions were encountered; (a) sustained ventricular tachyarrhythmias, (b) $>3 \mathrm{~s}$ asystole or 3rd degree atrio-ventricular (AV) block, (c) supraventricular arrhythmias with cardioembolic stroke, (d) worsening of heart failure needing treatment as an inpatient or needed cardiac transplantation, and (e) death from a cardiovascular cause.

\subsection{Statistical analysis}

The data are expressed as means \pm standard deviation (SD) for continuous variables and numbers and percentages for categorical variables. The possible role played by selected variables, including muscle weakness and gene mutations, in relationship with the presence of any cardiac event was examined by single variable analysis. Student's $t$-test was used for comparisons of normally distributed continuous variables, and chi-square or Fisher's exact test for differences in frequency, as appropriate. The incidence of all and of major cardiac AEs was analyzed, using the Kaplan-Meier method. All analyses were performed with 
the STATA $^{\circledR}$ statistical software, version 10.1 (StataCorp LP, College Station, TX).

\section{Results}

\subsection{Patient population}

We identified 28 patients (17 men, mean age $37.7 \pm$ 14.4 years), from 19 families, with $D E S$ mutations. Probands were patients who were referred to Neurology Units for the assessment of muscle symptoms. The genetic screening of probands' relatives displayed a $D E S$ mutation in 9 individuals, including 7 who had no prior history of muscle or cardiac disease, 1 with a previously undiagnosed myopathy, and 1 with a history of severe arrhythmia. A familial transmission of the disease with an autosomal dominant pattern was found in 16 independent index patients (with a total of 25 patients or mutation carriers). Familial genetic screening could not be performed to differentiate de novo mutations from autosomal dominant transmissions in 3 patients who presented with sporadic cardiac and muscle disease.

\subsection{Genetic analyses (Table 1, Fig. 1)}

Sequencing DES gene led to the identification of 18 different mutations in the 19 unrelated patients. Eleven mutations had already been described, while 7 are novel ones. The novel mutations consisted in 4 null mutations; a stop codon p.Lys144X, two splice sites mutations affecting exon $3 ;$ c. $640-2: \mathrm{A}>\mathrm{C}, \mathrm{c} .735+3: \mathrm{A}>\mathrm{G}$ and a mutation transforming the termination codon in a coding codon p.X471Tyr and thus giving rise to a putative longer protein. Four mutations resulted in substitution codons,

Table 1

DES mutations for each family studied.

\begin{tabular}{|c|c|c|c|c|}
\hline Family & Mutation & cDNA (NM_001927.3) & Mutation localisation & Mutation type \\
\hline 1 & p.Leu338Arg & c. $1013 \mathrm{~T}>\mathrm{G}$ & 2B helix & Missense \\
\hline 2 & p.Pro419Ser ${ }^{*}$ & c. $1255 \mathrm{C}>\mathrm{T}$ & Tail & Missense \\
\hline 3 & Putative exon 3 skipping ${ }^{*}$ & c. $640-2 \mathrm{~A}>\mathrm{C}$ & 1B helix & Splice \\
\hline 4 & p.Asp336 Tyr ${ }^{*}$ & c. $1006 \mathrm{G}>\mathrm{T}$ & 2B helix & Missense \\
\hline 5 & p.X471Tyr ${ }^{*}$ & c. $1413 \mathrm{~A}>\mathrm{C}$ & Tail & Abolition Ter codon \\
\hline 6 & p.Lys $144 X^{*}$ & c. $430 \mathrm{~A}>\mathrm{T}$ & 1B helix & Non sense \\
\hline 7 & p.Arg454Trp & c. $1360 \mathrm{C}>\mathrm{T}$ & Tail & Missense \\
\hline 8 & p.Glu245Asp or exon 3 skipping ${ }^{*}$ & c. $735 \mathrm{G}>\mathrm{T}$ & 1B helix & Missense or splice \\
\hline 9 & p.Arg406Trp & c. $1216 \mathrm{C}>\mathrm{T}$ & 2B helix & Missense \\
\hline 10 & p.Glu439Lys ${ }^{*}$ & c. $1315 \mathrm{G}>\mathrm{A}$ & Tail & Missense \\
\hline 11 & p.Ser2Ile & c. $.5 \mathrm{G}>\mathrm{T}$ & Head & Missense \\
\hline 12 & p.Ser2Ile & c. $5 \mathrm{G}>\mathrm{T}$ & Head & Missense \\
\hline 13 & p.Thr442Ile & c. $1325 \mathrm{C}>\mathrm{T}$ & Tail & Missense \\
\hline 14 & p.Asn342Asp & c. $1024 \mathrm{~A}>\mathrm{G}$ & 2B helix & Missense \\
\hline 15 & Putative exon 3 skipping & c. $735+3 \mathrm{~A}>\mathrm{G}$ & 1B helix & Splice \\
\hline 16 & p.Arg355Pro & c. $1064 \mathrm{G}>\mathrm{C}$ & 2B helix & Missense \\
\hline 17 & p.Arg454Trp & c. $1360 \mathrm{C}>\mathrm{T}$ & Tail & Missense \\
\hline 18 & p.Glu413Arg & c.1237_1238delinsAG & 2B helix & Missense \\
\hline 19 & p.Leu345Pro & c. $1034 \mathrm{~T}>\mathrm{C}$ & 2B helix & Missense \\
\hline
\end{tabular}

${ }^{*}$ New mutations.

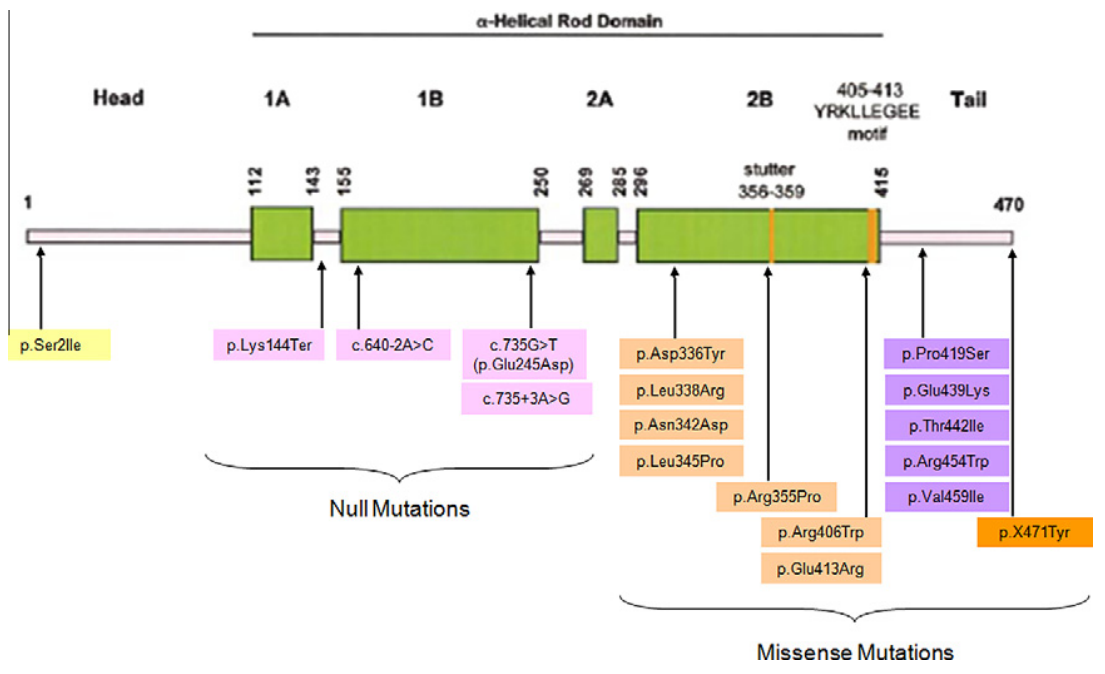

Fig. 1. Desmin mutations. 
p.Asp336Tyr, p.Pro419Ser, p.Glu439Lys. The spectrum of the 19 identified mutations, consisted in 14 missense mutations and 5 null alleles. Two mutations were of uncertain consequences without mRNA analysis, the c. $735 \mathrm{G}>\mathrm{T}$ on the last nucleotide of exon 3 that could lead either to p.Glu245Asp or to an aberrant splicing, and the c. $735+3: \mathrm{A}>\mathrm{G}$ which abolish the splice donor site of exon 3 .

\subsection{Baseline cardiac and skeletal muscular status (Tables 2 , $3 a$ and $3 b$ )}

Baseline cardiac and muscle assessments were completed in all patients.

Muscle involvement was identified at baseline evaluations in $21 / 28$ patients, including 19 probands in whom muscle

Table 2

Baseline characteristics of the 28 patients included in this analysis.

\begin{tabular}{|c|c|}
\hline $\begin{array}{l}\text { Age, y } \\
\text { Women }\end{array}$ & $\begin{array}{l}37.7 \pm 14.4 \\
11(39.2)\end{array}$ \\
\hline $\begin{array}{l}\text { Muscular assessment } \\
\text { Muscle weakness } \\
\text { Functional status: wheelchair bound/ambulatory } \\
\text { Muscle weakness topography: } \\
\text { - Proximal/distal/both } \\
\text { - Lower limbs/upper limbs/both } \\
\text { - Axial } \\
\text { - Facial }\end{array}$ & $\begin{array}{l}21(75.0) \\
3(10.7) / 18(57.1) \\
1(3.0) / 7 \\
(25.0) / 12(35.7) \\
1(3.0) / 2 \\
(7.0) / 18(57.1) \\
18(64.2) \\
6(21.4)\end{array}$ \\
\hline $\begin{array}{l}\text { Previous history of major cardiac adverse events } \\
\text { 3d degree AV block } \\
\text { Sustained ventricular tachycardia } \\
\text { Hospitalization for heart failure }\end{array}$ & $\begin{array}{l}7(25.0) \\
1(3.5) \\
2(7.0)\end{array}$ \\
\hline $\begin{array}{l}\text { Cardiac symptoms } \\
\text { Lightheadedness } \\
\text { Palpitations } \\
\text { Dyspnea }\end{array}$ & $\begin{array}{l}5(18.5) \\
9(33.3) \\
9(32.1)\end{array}$ \\
\hline $\begin{array}{l}\text { Electrocardiogram } \\
\text { Normal } \\
\text { 1st degree AV block, bundle branch block, or } \\
\quad \text { fascicular block } \\
\text { Bifascicular block/trifascicular block } \\
\text { Paced rhythm }\end{array}$ & $\begin{array}{l}3(10.7) \\
10(35.7) \\
5(17.8) / 4(14.2) \\
6(21.4)\end{array}$ \\
\hline $\begin{array}{l}\text { Echocardiography } \\
\text { Normal } \\
\text { Dilated/hypertrophic/restrictive cardiomyopathy }\end{array}$ & $\begin{array}{l}12(42.8) \\
3(10.7) / 8(28.5) / 1 \\
(3.5)\end{array}$ \\
\hline $\begin{array}{l}\text { Left ventricular systolic dysfunction } \\
\text { Right ventricular disease }\end{array}$ & $\begin{array}{l}5(17.8) \\
6(21.4)\end{array}$ \\
\hline $\begin{array}{l}\text { 24-h ambulatory electrocardiogram } \\
\text { Supraventricular arrhythmias } \\
\text { Ventricular arrhythmias } \\
\text { Sinus pauses }\end{array}$ & $\begin{array}{l}4(14.8) \\
1(3.7) \\
1(3.7)\end{array}$ \\
\hline $\begin{array}{l}\text { Treatment } \\
\text { Pacemaker } \\
\text { Implantable cardioverter defibrillator } \\
\text { Angiotensin-converting enzyme inhibitor } \\
\text { Beta-adrenergic blocker } \\
\text { Loop diuretic } \\
\text { Oral anticoagulant }\end{array}$ & $\begin{array}{l}10(35.7) \\
1(3.5) \\
7(25.0) \\
8(29.6) \\
4(14.8) \\
4(14.8)\end{array}$ \\
\hline
\end{tabular}

biopsy revealed myofibrillar myopathy, 1 relative with a previously undiagnosed myopathy, and 1 relative with mild asymptomatic weakness on physical examination.

Cardiac involvement was present in $27 / 28$ patients, including isolated or combined conduction abnormalities in 25 , supraventricular arrhythmias in 4, ventricular arrhythmias in 2 , and myocardial disease in 14 . Thirteen patients complained of symptoms suggestive of underlying heart or respiratory disease such as dyspnea, syncope, palpitations or lightheadedness. Nine patients had suffered from $\geqslant 1$ major cardiac AEs 1-17 years prior to baseline evaluation. Among the 9 relatives of probands carrying a $D E S$ mutation, previously undiagnosed cardiac disease was identified in 6 , including 4 who had no muscle involvement. In addition, from family history, we identified 16 additional first- or second-degree relatives among 9 families who had histories of complete AV block, sustained ventricular tachyarrhythmia, or sudden death (no. 2, 4, 10-11, 13-15, 17, 19) and could not undergo genetic testing. Advanced conduction system disease requiring permanent pacing was present in 14 relatives, of whom 6 died suddenly.

\subsection{Long-term clinical outcomes (Fig. 2, additional material)}

The average follow-up, complete in all but 2 (no. 4-1 and 13-2) of the 28 patients, was $10.4 \pm 9.4$ years. Patient

Table $3 \mathrm{a}$

Individual baseline characteristics.

\begin{tabular}{|c|c|c|c|c|c|}
\hline $\begin{array}{l}\text { Family- } \\
\text { patient }\end{array}$ & Sex & Age & $\begin{array}{l}\text { Muscle } \\
\text { involvement }\end{array}$ & $\mathrm{CK}^{* *}$ & $\begin{array}{l}D E S \\
\text { mutations }\end{array}$ \\
\hline $1-1^{*}$ & M & 54 & $\mathrm{Y}$ & 4 & p.Leu338Arg \\
\hline $2-1^{*}$ & $\mathrm{~F}$ & 33 & $\mathrm{Y}$ & NL & p.Pro419Ser \\
\hline $2-2$ & M & 50 & $\mathrm{Y}$ & 1.3 & p.Pro419Ser \\
\hline $2-3$ & $\mathrm{~F}$ & 71 & $\mathrm{~N}$ & NL & p.Pro419Ser \\
\hline $3-1^{*}$ & $\mathrm{~F}$ & 33 & $\mathrm{Y}$ & NL & c. $640-2 \mathrm{~A}>\mathrm{C}$ \\
\hline $3-2$ & $\mathrm{~F}$ & 27 & $\mathrm{~N}$ & NL & c. $640-2 \mathrm{~A}>\mathrm{C}$ \\
\hline $4-1^{*}$ & M & 38 & $\mathrm{Y}$ & 4 & p.Asp336Tyr \\
\hline $5-1^{*}$ & M & 36 & $\mathrm{Y}$ & NL & p.X471Tyr \\
\hline $6-1^{*}$ & $\mathrm{~F}$ & 44 & $\mathrm{Y}$ & 5 & p.Lys $144 X$ \\
\hline $6-2$ & M & 74 & $\mathrm{~N}$ & NL & p.Lys $144 X$ \\
\hline $7-1^{*}$ & M & 25 & $\mathrm{Y}$ & 4.5 & p.Arg454Trp \\
\hline $8-1^{*}$ & $\mathrm{~F}$ & 33 & $\mathrm{Y}$ & 1.1 & p.Glu245Asp \\
\hline $8-2$ & $\mathrm{~F}$ & 9 & $\mathrm{~N}$ & NL & p.Glu245Asp \\
\hline $9-1^{*}$ & $\mathrm{~F}$ & 31 & $\mathrm{Y}$ & 1.05 & p.Arg406Trp \\
\hline $10-1^{*}$ & M & 54 & $\mathrm{Y}$ & 1.5 & p.Glu439Lys \\
\hline $10-2$ & M & 28 & $\mathrm{~N}$ & NL & p.Glu439Lys \\
\hline $11-1^{*}$ & M & 49 & $\mathrm{Y}$ & 1.8 & p.Ser2Ile \\
\hline $12-1^{*}$ & M & 44 & $\mathrm{Y}$ & 2.9 & p.Ser2Ile \\
\hline $13-1^{*}$ & $\mathrm{~F}$ & 44 & $\mathrm{Y}$ & $\mathrm{NL}$ & p.Thr442Ile \\
\hline $13-2$ & $\mathrm{~F}$ & 27 & $\mathrm{~N}$ & 1.4 & p.Thr442Ile \\
\hline $13-3$ & M & 36 & $\mathrm{Y}$ & 3.1 & p.Thr442Ile \\
\hline $14-1^{*}$ & M & 48 & Y & 2.7 & p.Asn $342 A s p$ \\
\hline $15-1^{*}$ & M & 29 & $\mathrm{~N}$ & NL & c. $735+3 \mathrm{~A}>\mathrm{G}$ \\
\hline $16-1^{*}$ & M & 29 & $\mathrm{Y}$ & 5 & p.Arg355Pro \\
\hline $17-1^{*}$ & M & 27 & $\mathrm{Y}$ & 1.3 & p.Arg454Trp \\
\hline $18-1^{*}$ & $\mathrm{~F}$ & 30 & $\mathrm{Y}$ & NL & p.Glu413Arg \\
\hline $18-2$ & M & 16 & $\mathrm{Y}$ & NL & p.Glu413Arg \\
\hline $19-1^{*}$ & M & 33 & $\mathrm{Y}$ & 2.9 & p.Leu345Pro \\
\hline
\end{tabular}


Table $3 b$

Individual baseline cardiac investigations.

\begin{tabular}{|c|c|c|c|c|c|c|c|c|}
\hline \multirow{2}{*}{$\begin{array}{l}\text { Fam- } \\
\mathrm{Pt}\end{array}$} & \multirow[t]{2}{*}{ History of cardiac disease (age) } & \multirow[t]{2}{*}{ Cardiac symptoms } & \multirow[t]{2}{*}{ ECG } & \multirow{2}{*}{$\begin{array}{l}\text { 24-h ambulatory } \\
\text { ECG }\end{array}$} & \multicolumn{3}{|l|}{ Echocardiography } & \multirow[t]{2}{*}{ Treatment } \\
\hline & & & & & Cardiomyopathy & LVEF & $\begin{array}{l}\text { RV } \\
\text { disease }\end{array}$ & \\
\hline $1-1^{*}$ & - & - & $\begin{array}{l}\text { Bifascicular } \\
\text { block }\end{array}$ & - & - & 0.68 & $\mathrm{~N}$ & - \\
\hline $2-1^{*}$ & - & Palpitations & $\begin{array}{l}\text { Bifascicular } \\
\text { block }\end{array}$ & - & - & 0.65 & $\mathrm{~N}$ & - \\
\hline $2-2$ & - & $\begin{array}{l}\text { Lightheadedness, } \\
\text { palpitations }\end{array}$ & $\begin{array}{l}\text { Bifascicular } \\
\text { block }\end{array}$ & - & Dilated & 0.45 & $\mathrm{~N}$ & Pacemaker \\
\hline $2-3$ & - & $\begin{array}{l}\text { Lightheadedness, } \\
\text { palpitations }\end{array}$ & $\begin{array}{l}\text { Trifascicular } \\
\text { block }\end{array}$ & - & Hypertrophic & 0.63 & $\mathrm{~N}$ & Pacemaker \\
\hline $3-1^{*}$ & 3rd degree AVB (32) & - & $\begin{array}{l}\text { Paced } \\
\text { rhythm }\end{array}$ & - & - & 0.62 & $\mathrm{~N}$ & Pacemaker \\
\hline $3-2$ & 3rd degree AVB (26) & - & $\begin{array}{l}\text { Paced } \\
\text { rhythm }\end{array}$ & - & - & 0.70 & $\mathrm{~N}$ & Pacemaker \\
\hline $4-1^{*}$ & 3rd degree AVB (37) & Dyspnea & $\begin{array}{l}\text { Trifascicular } \\
\text { block }\end{array}$ & - & Dilated & 0.45 & $\mathrm{~N}$ & Pacemaker \\
\hline $5-1^{*}$ & 3rd degree AVB (35) & - & $\begin{array}{l}\text { Paced } \\
\text { rhythm }\end{array}$ & - & - & 0.65 & $\mathrm{~N}$ & Pacemaker \\
\hline $6-1^{*}$ & NSVT (37) & Dyspnea & LBBB & NSVT & Dilated & 0.4 & $\mathrm{~N}$ & - \\
\hline $6-2$ & - & - & - & & Hypertrophic & 0.65 & $\mathrm{Y}$ & - \\
\hline $7-1^{*}$ & Hospitalization for HF (19) & Dyspnea & - & - & Hypertrophic & 0.20 & $\mathrm{Y}$ & - \\
\hline $8-1^{*}$ & 3rd degree AVB (23) & - & $\begin{array}{l}\text { Paced } \\
\text { rhythm }\end{array}$ & - & Hypertrophic & 0.68 & $\mathrm{Y}$ & Pacemaker \\
\hline $8-2$ & - & Palpitations & RBBB & Atrial flutter & - & 0.65 & $\mathrm{~N}$ & - \\
\hline $9-1^{*}$ & 3rd degree AVB (14) & - & $\begin{array}{l}\text { Paced } \\
\text { rhythm }\end{array}$ & Atrial fibrillation & - & 0.65 & $\mathrm{~N}$ & Pacemaker \\
\hline $10-1^{*}$ & Atrial fibrillation (51) & - & $\begin{array}{l}\text { Trifascicular } \\
\text { block }\end{array}$ & - & - & 0.65 & $\mathrm{Y}$ & - \\
\hline $10-2$ & - & - & $\mathrm{RBBB}$ & - & - & 0.69 & $\mathrm{~N}$ & - \\
\hline $11-1^{*}$ & - & Palpitations & $\begin{array}{l}\text { Bifascicular } \\
\text { block }\end{array}$ & - & Hypertrophic & 0.70 & $\mathrm{~N}$ & - \\
\hline $12-1^{*}$ & - & - & LPFB & - & Hypertrophic & 0.69 & $\mathrm{~N}$ & - \\
\hline $13-1^{*}$ & - & - & LBBB & - & - & 0.68 & $\mathrm{~N}$ & - \\
\hline $13-2$ & - & - & - & - & - & 0.65 & $\mathrm{~N}$ & - \\
\hline $13-3$ & - & Palpitations & $\mathrm{RBBB}$ & - & - & 0.57 & $\mathrm{~N}$ & - \\
\hline $14-1^{*}$ & - & Lightheadedness & 1st degree AVB & Sinuspauses & - & 0.62 & $\mathrm{~N}$ & Pacemaker \\
\hline $15-1^{*}$ & & $\begin{array}{l}\text { Lightheadedness, } \\
\text { palpitations }\end{array}$ & $\begin{array}{l}\text { Trifascicular } \\
\text { block }\end{array}$ & - & Hypertrophic & 0.70 & $\mathrm{~N}$ & Pacemaker \\
\hline $16-1^{*}$ & - & - & $\begin{array}{l}\text { Bifascicular } \\
\text { block }\end{array}$ & - & - & 0.65 & $\mathrm{Y}$ & - \\
\hline $17-1^{*}$ & $\begin{array}{l}\text { Atrial fibrillation, SVT, } \\
\text { 3rd degree AVB, } \\
\text { Hospitalization for HF (24) }\end{array}$ & Dyspnea, syncope & $\begin{array}{l}\text { Atrial } \\
\text { fibrillation, } \\
\text { paced rhythm }\end{array}$ & - & Restrictive & 0.30 & $\mathrm{Y}$ & $\mathrm{ICD}$ \\
\hline $18-1^{*}$ & - & $\begin{array}{l}\text { Lightheadedness, } \\
\text { palpitations }\end{array}$ & LAFB & - & - & 0.65 & $\mathrm{~N}$ & - \\
\hline $18-2$ & - & - & LBBB & - & Hypertrophic & 0.65 & $\mathrm{~N}$ & - \\
\hline $19-1^{*}$ & - & - & RBBB & - & - & ND & $\mathrm{N}$ & - \\
\hline
\end{tabular}

* Probands.

4-1 was lost to follow-up after year 2007, and patient 13-2 was alive at the end of the study, according to the National Death Index.

Five patients died at a mean age of $58.0 \pm 6.5$ years, representing a $17.8 \%$ mortality rate, including 3 for whom cardiac complications were the primary cause of death (age $=60.3 \pm 5.5)$ : Patient $13-1$ died suddenly 18 years after the implantation of a pacemaker, which could not be interrogated post mortem; Patients 10-1 and 13-3 died of end stage heart failure due to dilated cardiomyopathy with a contraindication to cardiac transplantation because of severe respiratory insufficiency. In addition, 2 patients died due to iatrogenic complications of cardiac treatment: Patient 7-1 who received immunosuppressive drugs after cardiac transplantation died from lymphoma with pulmonary infection; Patient 11-1 died of hemorrhagic stroke on oral anticoagulants for atrial fibrillation.

Major cardiac AEs developed in 13/28 patients, including high degree conduction defects requiring permanent pacing in 8 , sustained ventricular tachycardia in 1 , hospitalizations 


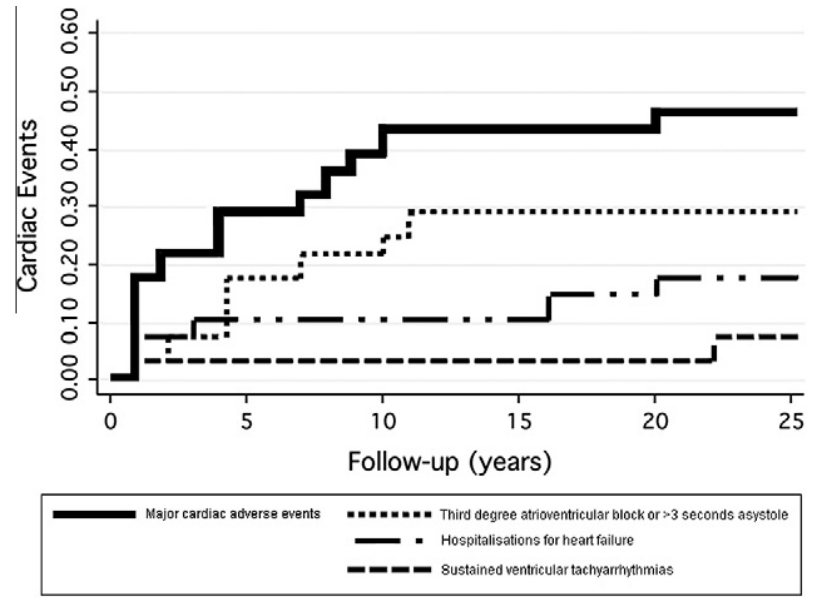

Fig. 2. Cumulative incidence of major cardiac adverse events in the 28 patients included.

for heart failure in 7, and cardioembolic stroke in 1. Seven out of the thirteen patients had no cardiac symptom at baseline.

Among the 19 patients with conduction blocks on the ECG at baseline and no prior history of major conduction defects, 8 developed high degree conduction disease, after a mean follow-up of 6 years, whereas no patient with normal ECG developed such complication. High degree blocks became apparent as syncope or decompensation of heart failure and required the implantation of a pacemaker in emergency. Pacemakers were replaced by implantable cardioverter defibrillators in 3 patients who developed sustained ventricular tachyarrhythmias, or who had family history of sudden death despite the implantation of a pacemaker.

Heart failure decompensation requiring treatment as inpatients occurred in 7 patients, 3 of whom underwent cardiac transplantation and 2 died. Among these 7 patients with severe heart failure, the cardiomyopathy patterns displayed by echocardiography were restrictive in 1, hypertrophic in 1, and dilated in 5. Echocardiography at baseline was normal in 4 out of these 7 patients.

Three out of the 9 probands' relatives carrying $D E S$ mutation developed major cardiac AEs, including 1 patient who died due to heart failure.

\subsection{Occurrence of muscle and cardiac involvement over lifetime}

The mean age at onset of disease was $31.7 \pm 13.3$ years. The first manifestations of the disease were muscle weakness in 11 patients who all developed cardiac involvement after $6.3 \pm 3.5$ years and cardiac involvement in 16 patients, 10 of whom developed muscle disease after $6.8 \pm 4.1$ years. Among the patients with a cardiac onset, 6 presented with major cardiac complications, including a 14-year old patient who underwent a pacemaker implantation for syncope due to 3rd degree AV block. Another patient presented at 8 years with atrial flutter revealed by palpitations.

\subsection{Correlations between clinical presentation and genetic characteristics}

No correlations were found between cardiac involvement, and muscle phenotype or genetic characteristics, including location of the mutation in the protein (head, $1 \mathrm{~B}$ and 2B helix domains, or tail) and type of mutation (nonsense, missense or splice).

\section{Discussion}

The main observations made in our study were (1) a high cardiac mortality and morbidity in DES mutations carriers, and (2) the absence of correlations between genotype and phenotype or skeletal and cardiac muscle involvement. The strengths of our study are (1) the number of patients included, (2) the standardized protocol of investigations, and (3) the mean 10-year duration of follow-up.

We found in this population suffering from desminopathy cardiac involvement in 27 and muscle involvement in $21 / 28$ of patients. Life-threatening cardiac complications were observed in 13 patients, at a mean age of 40 years, including sudden death, endstage heart failure, AV block and syncope requiring permanent pacing, and sustained ventricular tachycardia. These complications were the first manifestation of cardiac involvement in $1 / 3$ of patients and were also observed among relatives carrying DES mutation without muscle involvement, including asymptomatic patients. In addition, genetic analyses, muscle studies, or type of cardiomyopathy did not predict the development of advanced cardiac disease. Therefore, the severity and unpredictability of cardiac complications suggest that cardiac investigations should be systematically and regularly repeated in all DES mutation carriers with a view to diagnose cardiac involvement as early as possible, including those without muscle involvement. Mutations in other genes than $D E S$, such as $L M N A$, might be present and responsible for the muscular and cardiac abnormalities in some of our patients but we have no data that may hardly support this hypothesis. The youngest patient in whom major cardiac AEs were identified was 14 years of age, suggesting the need to begin meticulous ECG and echocardiographic surveillance no later than in adolescence.

The 18 different variants that we found were spanning all along the gene and consequently the protein. The mutation spectrum seems to show that the null mutations were preferentially located on the N-terminal domain of the protein (Domain 1A and 1B helix domain) putatively leading to an absence of the mutant allele except the mutation abolishing the termination codon. Only 1 missense mutation (p.Ser2Ile) was located in the head domain. All other substitutions acting as a dominant negative effect were located in the $2 \mathrm{~B}$ helix and tail domains of the protein $(7$ in the $2 \mathrm{~B}$ helix, and 5 in the tail domain). This extensive location of mutations does not facilitate the correlations studies between the genotype and the phenotype and no particular correlation between mutation types or locations 
and skeletal muscle or cardiac disease could emerge from this work.

Conduction system disease was the most frequent manifestation of the disease. Conduction abnormalities were overall present in 25/28, and severe symptomatic abnormalities of impulse propagation requiring permanent pacing in nearly 16/28 of patients. Among patients presenting at baseline with 1st degree AV block, fascicular block, or both, around $40 \%$ developed a higher degree of blocking, after mean follow-up of 6.0 years. This suggests that the implantation of a pacemaker should be systematically discussed with patients whose ECG reveals the presence of conduction abnormalities even in the absence of any symptom.

Ventricular arrhythmias as a common manifestation of cardiac involvement have not been reported so far in DES mutated patients but a single case report has already been published underlying this occurrence [16]. Ventricular arrhythmias were observed in 4 out of our patients, including 2 who developed hemodynamically unstable, and sustained ventricular tachycardia. In addition, sudden death occurred in 1 another patient and in 4 first-degree relatives who received permanent pacemakers for severe conduction defects. However, in those cases, the devices' memories could not be interrogated to confirm ventricular arrhythmias. No significant association could be drawn between ventricular arrhythmias, myocardial disease, and ventricular function, suggesting that ventricular arrhythmias are implicated in the sudden death of $D E S$ mutated patients regardless of ventricular function. In these cases, it appears that cardioverter defibrillator is a more usefulness tool in primary prevention than pacemaker but this could not be statistically proven.

The other prominent cause of morbidity and mortality in our patients was myocardial disease, as 8 out of our 28 patients were hospitalized for treatment of heart failure, and 5 developed end stage heart failure. Severe right ventricular, and left ventricular myocardial involvement have been observed, such a severe phenotype having also reported earlier in 1 member of a large family [15]. Our series of patients also document dilated, hypertrophic and restrictive cardiomyopathies as the worsening factors in heart failure, but statistical analyses did not identify any correlation between type of cardiomyopathy and need for hospitalization. Cardiac transplantation was performed in 3 of those patients, who had mild muscle weakness and no respiratory insufficiency. Absence of complications specifically related to the genetic disease during follow-up, as previously reported [17], confirmed that cardiac transplantation is a valuable therapeutic option in patients who have developed end stage heart failure due to desminopathy.

\subsection{Study limitations}

Despite being the largest series of patients presenting with desminopathy reported so far, the number of patients may have been insufficient to reach sufficient statistical power to identify genotype- phenotype correlations.

\section{Conclusions}

Patients carrying DES mutations presenting with myofibrillar myopathies or without muscle involvement, are at high risk of developing major cardiac complications, including high-degree AV block, ventricular tachycardia and end stage heart failure. In these patients cardiac investigations are suggested to be performed, at the time of early diagnosis and at a yearly basis during follow-up. Patient's relative who could potentially carry $D E S$ mutation should also be offered to the same work-ups even if muscle involvement is absent. The prophylactic implantation of a pacemaker or a defibrillator should systematically be discussed in patients presenting with conduction system disease.

\section{Study funding}

This study was funded by grants from the Association Française contre les Myopathies (AFM) and Assistance Publique-Hôpitaux de Paris (Myology consultation, molecular analyses)

\section{Acknowledgements}

The authors would like to express special thanks to Drs Alain Delabays and Xavier Jeanrenaud (Service de Cardiologie, Centre Hospitalier Universitaire Vaudois, Lausanne) who helped greatly in giving the results of several cardiac studies. As they contributed equally to this work, Karim Wahbi and Anthony Béhin, as well as Thierry Kuntzer and Denis Duboc, are respectively two first- and last co-author of this article.

\section{References}

[1] Lazarides E. Intermediate filaments as mechanical integrators of cellular space. Nature 1980;238:249-56.

[2] Goldfarb LG, Park KY, Cervenáková L, et al. Missense mutations in desmin associated with familial cardiac and skeletal myopathy. Nat Genet 1998;19:402-3.

[3] Dalakas MC, Park KY, Semino-Mora C, Lee HS, Sivakumar K, Goldfarb LG. Desmin myopathy a skeletal myopathy with cardiomyopathy caused by mutations in the desmin gene. N Engl J Med 2000;342:770-80.

[4] Li D, Tapscoft T, Gonzalez O, et al. Desmin mutation responsible for idiopathic dilated cardiomyopathy. Circulation 1999;100:461-4.

[5] Goudeau B, Dagvadorj A, Rodrigues-Lima F, et al. Structural and functional analysis of a new desmin variant causing desmin-related myopathy. Hum Mutat 2001;18:388-96.

[6] Miyamoto Y, Akita H, Shiga N, et al. Frequency and clinical characteristics of dilated cardiomyopathy caused by desmin gene mutation in a Japanese population. Eur Heart J 2001;22:2284-9.

[7] Taylor MR, Slavov D, Ku L, et al. Prevalence of desmin mutations in dilated cardiomyopathy. Circulation 2007;115:1244-51.

[8] Selcen D, Ohno K, Engel AG. Myofibrillar myopathy: clinical, morphological and genetic studies in 63 patients. Brain 2004;127:439-51.

[9] Olivé M, Goldfarb L, Moreno D, et al. Desmin-related myopathy: clinical, electrophysiological, radiological, neuropathological and genetic studies. J Neurol Sci 2004;219:125-37. 
[10] Arbustini E, Pasotti M, Pilotto A, et al. Desmin accumulation restrictive cardiomyopathy and atrioventricular block associated with desmin gene defects. Eur J Heart Fail 2006;8:477-83.

[11] Pruszczyk P, Kostera-Pruszczyk A, Shatunov A, et al. Restrictive cardiomyopathy with atrioventricular conduction block resulting from a desmin mutation. Int J Cardiol 2007;117:244-53.

[12] Kostera-Pruszczyk A, Pruszczyk P, Kaminska A, Lee Hee-Suk, Goldfarb LG. Diversity of cardiomyopathy phenotypes caused by mutations in desmin. Int J Cardiol 2008;131:146-7.

[13] Strach K, Sommer T, Grohé C, et al. Clinical, genetic, and cardiac magnetic resonance imaging findings in primary desminopathy. Neuromuscul Disord 2008;18:475-82.

[14] Cheitlin MD, Armstrong WF, Aurigemma GP, et al. Guideline Update for the Clinical Application of Echocardiography: summary article A report of the American College of Cardiology/American Heart Association Task Force on Practice Guidelines. J Am Soc Echocardiogr 2003;16:1091-110.

[15] van Tintelen JP, Van Gelder IC, Asimaki A, et al. Severe cardiac phenotype with right ventricular predominance in a large cohort of patients with a single missense mutation in the DES gene. Heart Rhythm 2009;6:1574-83.

[16] Luethje LG, Boennemann C, Goldfarb L, Goebel HH, Halle M. Prophylactic implantable cardioverter defibrillator placement in a sporadic desmin related myopathy and cardiomyopathy. Pacing Clin Electrophysiol 2004;27:559-60.

[17] Rees W, Schüler S, Hummel M, Hetzer R. Heart transplantation in patients with muscular dystrophy associated with end-stage cardiomyopathy. J Heart Lung Transplant 1993;12:804-7. 